\title{
Osteoblastoma agresivo del hueso grande
}

\author{
A. Ortega Briones ${ }^{(1)}$, A. García Olea ${ }^{(1)}$, A. Crespo Rodríguez ${ }^{(2)}$ \\ Hospital Universitario Puerta de Hierro Majadahonda, Madrid. \\ (1) Médico Adjunto Cirugía Ortopédica y TRaumatología \\ (2) Médico Adjunto Radiología
}

Correspondencia:

Dra. Alina Ortega Briones

Hospital Universitario Puerta de Hierro Majadahonda

C/ Manuel de Falla, 1

28222 Majadahonda, Madrid

Teléfono: 911916000

e-mail: alina.ob@gmail.com

\begin{abstract}
Presentamos el caso de un paciente diagnosticado de osteoblastoma del hueso grande tratado de manera satisfactoria mediante legrado y relleno de autoinjerto. La incidencia de este tipo de tumores es rara en los huesos de la mano y es de suma importancia realizar un diagnóstico diferencial tanto radiológico como anatomopatológico. Las opciones de tratamiento incluyen el legrado o curetaje y posterior relleno de injerto, la resección en bloque, y la utilización de un injerto óseo vascularizado. El retraso del diagnóstico, y en consecuencia del tratamiento, supone la necesidad de utilizar técnicas más agresivas, y por tanto más incapacitantes funcionalmente.
\end{abstract}

Palabras clave: osteoblastoma, mano, hueso grande.
We present the case of a patient diagnosed of osteoblastoma of the capitate bone successfully treated by curettage and autogenous bone graft. This type of tumour is uncommon in the bones of the hand and, in these cases, it is very important to perform radiological and histological differential diagnoses. Treatment options include scraping or curettage and subsequent autogenous bone graft, or resection en bloc, as well as more aggressive techniques using vascularised bone grafts. Delayed diagnosis and the type of treatment, often partial arthrodesis, can cause severe disability.

Key words: osteoblastoma, hand, capitate bone.

\section{OBJETIVO}

S e presenta el caso de un paciente varón de 20 años, que consultó por dolor en inflamación progresiva e inespecífica en la muñeca derecha, debida a un osteoblastoma localizado en el hueso grande. El diagnóstico se realizó mediante biopsia.

\section{INTRODUCCIÓN}

El osteoblastoma es una lesión poco frecuente, representa menos del $1 \%$ de la totalidad de los tumores óseos benignos ${ }^{1}$. Fue descrito inicialmente por Dalhin ${ }^{2}$ y Johnson en 1954, quienes definieron la lesión como «osteoma osteoide gigante». En 1956 Liechtenstein $^{3}$ y Jaffe ${ }^{4}$ propusieron el término osteoblastoma benigno.

Presenta múltiples semejanzas anatomopatológicas y radioclínicas con el osteoma osteoide. La distinción entre ellos se fundamenta en características epidemiológicas, radioclínicas, evolutivas e histológicas.

El osteoblastoma predomina en el sexo masculino (2:1) entre los 10 y 30 años. Su localización es característica en la columna vertebral 


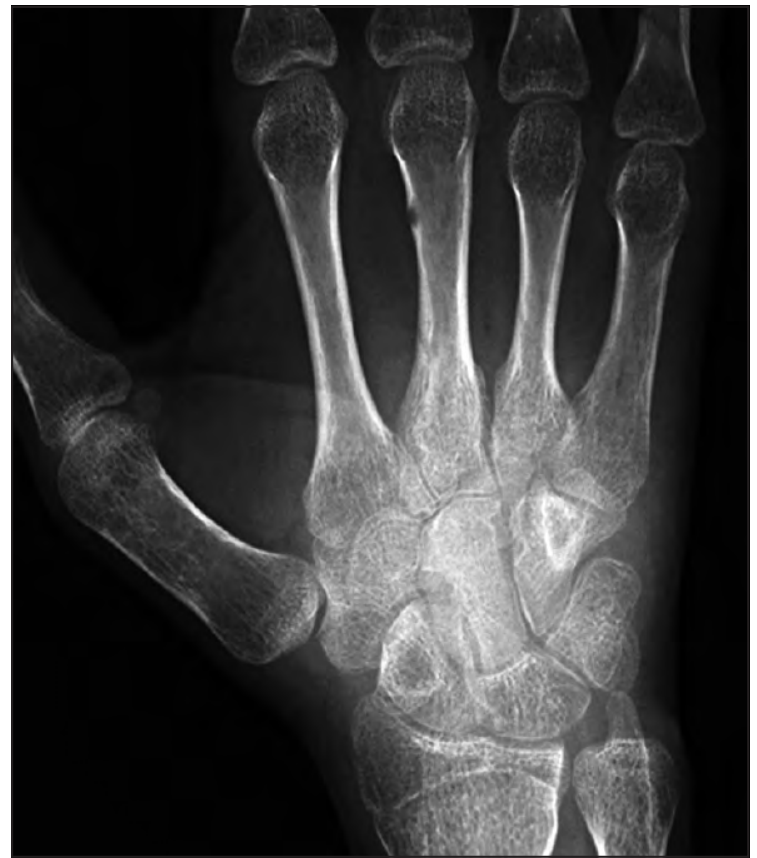

Figura 1. Radiografía AP de la muñeca derecha. Lesión quística en hueso grande.

(40\%), pelvis, en los huesos largos de los miembros y en los huesos del pie. Su presencia en los huesos del carpo es muy poco frecuente ${ }^{5}$ por lo que es decisivo realizar un diagnóstico anatomopatológico preciso.

\section{CASO CLÍNICO}

Paciente varón de 20 años, diestro, sin antecedentes médico-quirúrgicos de interés. Realiza terapia de manualidades por retraso psicomotor leve.

Acude a consultas de traumatología por dolor en muñeca derecha de más de 2 años y evolución progresiva a mayor intensidad. Se solicitan radiografías convencionales de muñeca que son anodinas. En la exploración física se describe leve aumento de temperatura y rubor, además de dolor a la palpación en dorso de carpo. El paciente fue diagnosticado inicialmente de síndrome de dolor regional complejo, por lo que se remite a tratamiento de Rehabilitación.

A los 9 meses el paciente es valorado en consultas de la Unidad de Mano por fracaso del tratamiento y aumento de la sintomatología, con la siguiente exploración física: dolor, tumefacción

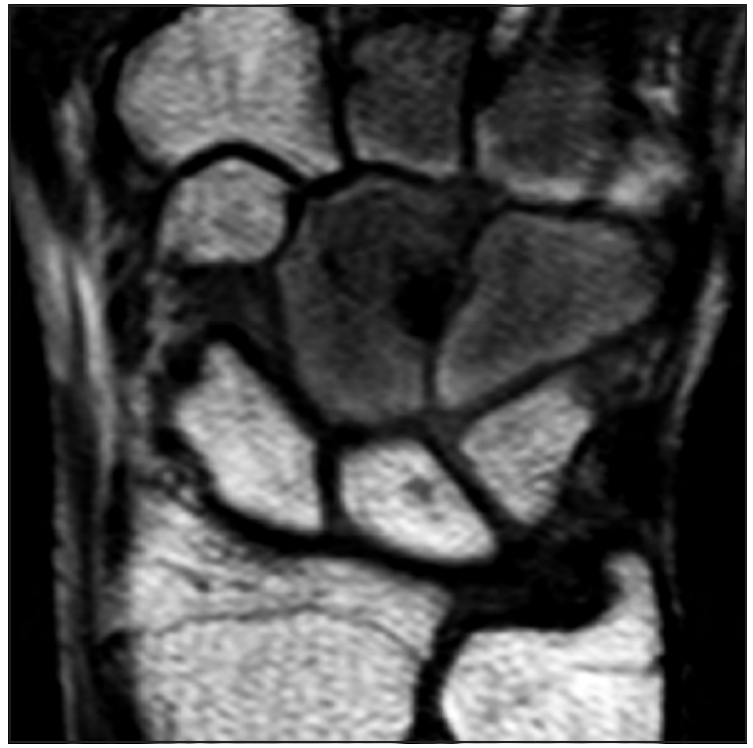

Figura 2. Imagen de RM potenciada en T1 de orientación coronal de la muñeca derecha muestra reemplazamiento de la señal medular ósea normal (grasa) del hueso grande y del ganchoso, así como de la base del tercer y cuarto metacarpianos. En el hueso grande se define una lesión focal marcadamente hipointensa, de localización excéntrica y adyacente a la carilla articular con el hueso ganchoso.

dura, calor y rubor en dorso de hueso grande y base de metacarpianos de los dedos medio y anular. Importante limitación para la de flexión y extensión por dolor.

Se solicitan nuevas pruebas de imagen. En la radiografía de muñeca (Figura 1) se observa una lesión quística, por lo que se solicita RM donde se objetiva lesión focal ósea en el hueso grande y reemplazamiento de la señal medular ósea de los huesos grande y ganchoso así como de la base de los metacarpianos tercero y cuarto, consistente en isointensidad de señal en T1 (Figura 2) e hiperintensidad en STIR (Figura 3) que se interpreta como edema óseo. El radiólogo no encuentra otros signos en las pruebas de imagen que permitan aquilatar el diagnóstico de la tumoración y aconseja realizar TC (Figuras $4 \mathbf{y}$ 5) que confirma la presencia de una lesión lítica bien definida con un comportamiento expansivo y evidencia de disrupción cortical tanto en el dorso como en la carilla articular con el hueso ganchoso. Finalmente se informa de posible sinovitis villonodular.

Se completa el estudio de imagen con gammagrafía ósea $\mathrm{Tc}^{99}$ donde se aprecia intenso de- 


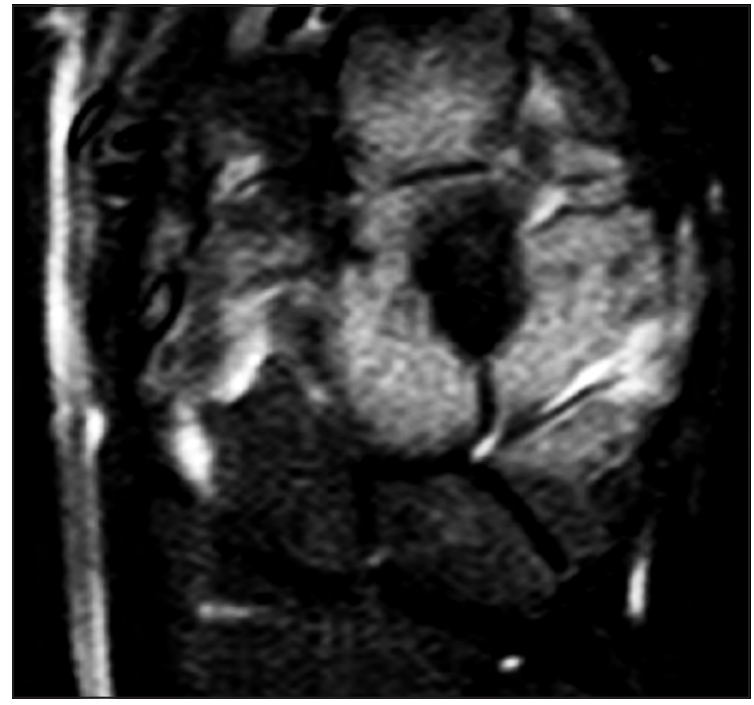

Figura 3. Imagen de RM potenciada en STIR de orientación coronal de la muñeca derecha, la lesión focal del hueso grande presenta una marcada hipointensidad de la señal. El hueso grande, el ganchoso, así como de la base del tercer y cuarto metacarpianos muestran hiperintensidad de la señal medular ósea en probable relación con edema óseo.

pósito en huesos carpianos derechos acompañados de fenómenos inflamatorios.

Se realizó punción aspiración con aguja fina (PAAF) siendo la muestra insuficiente, por lo que se decidió realizar artrotomía y biopsia intraoperatoria mediante abordaje dorsal del carpo.

Al realizar la artrotomía hallamos una sinovial hipertrófica, oscura y friable que parece penetrar en hueso. Se solicitó biopsia intraoperatoria, informada como sinovitis villonodular pigmentada, sin malignidad y con bordes bien definidos. Dado que el hueso grande presentaba una amplia rotura en su cara articular con el ganchoso y éste también parecía estar roto, se realizó curetaje, relleno con injerto de cresta iliaca y artrodesis grande-ganchoso con 2 tornillos miniAcutrak $^{\mathrm{tm}}$. El informe anatomopatológico posterior determina que la lesión implica fragmentos óseos compatibles con osteoblastoma. Éste dato, unido a la presencia de tejido fibroso perilesional intensamente vascularizado reafirma el diagnóstico de osteoblastoma del hueso grande.

El paciente realiza controles clínico-radiológicos cada 9 meses y a los 2 años de la cirugía no se constata recidiva de sintomatología o radiología (Figura 6).

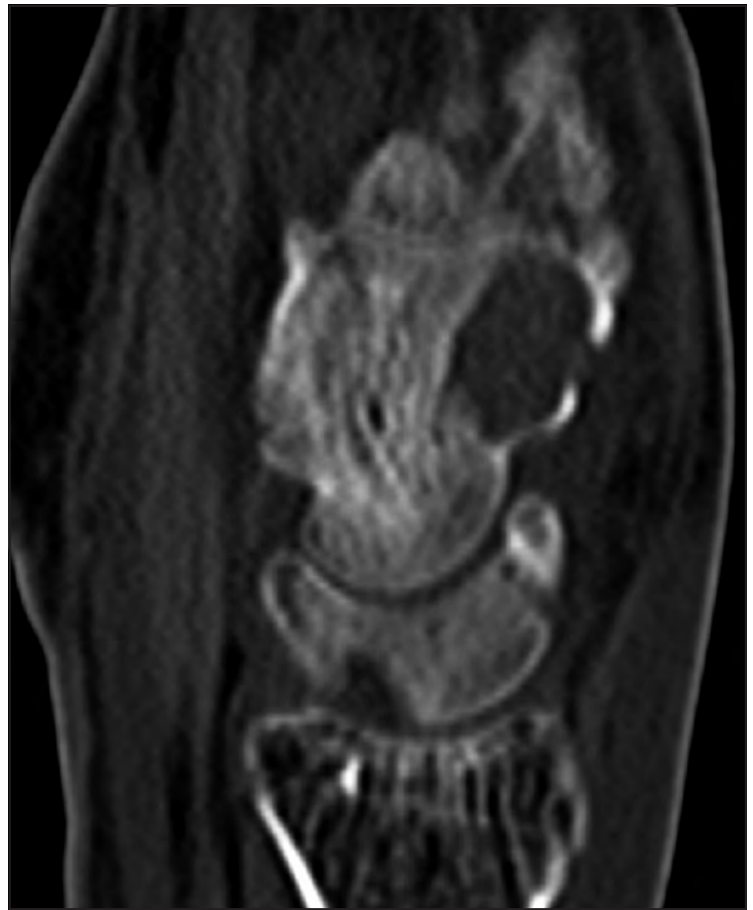

Figura 4. Imagen de TC de la muñeca derecha, en algoritmo óseo y de orientación sagital que muestra una lesión geográfica lítica en el hueso grande y la disrupción cortical en la superficie dorsal.

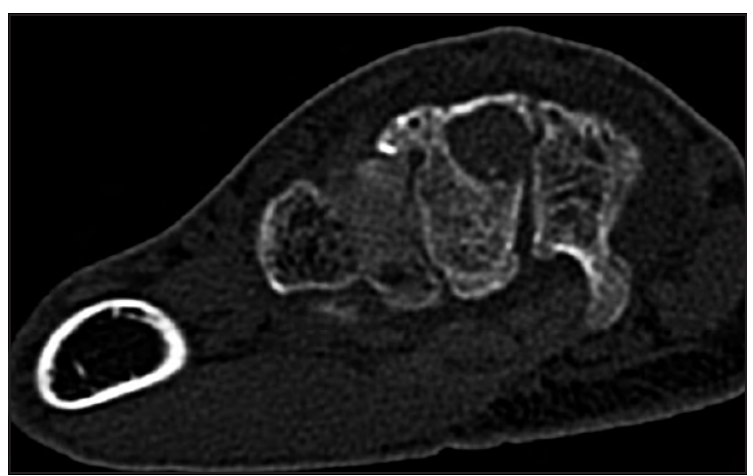

Figura 5. Imagen de TC de la muñeca derecha, en algoritmo óseo y de orientación axial que muestra la lesión focal lítica en el hueso grande con una calcificación puntiforme en declive así como disrupción cortical en la superficie articular del hueso grande con el ganchoso. Dicha articulación presenta además irregularidad de las superficies articulares y pequeña geoda subcondral en el hueso ganchoso.

\section{DISCUSIÓN}

Los tumores óseos son muy raros en carpo, y son en su mayoría benignos.

La localización más habitual del osteoblastoma es la columna vertebral (40\%), seguida de la 


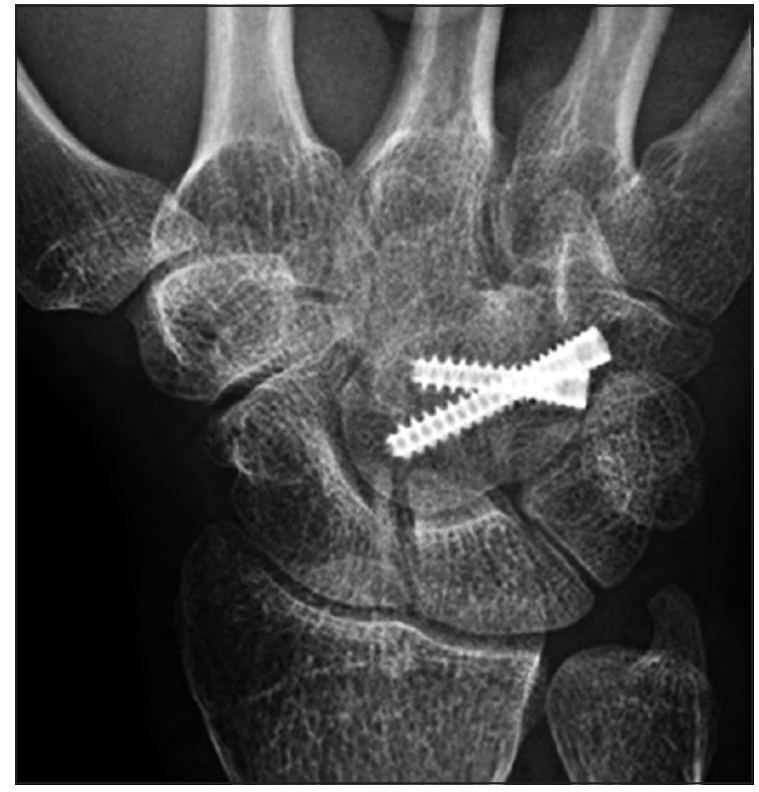

Figura 6. Radiografía AP de muñeca, control radiológico a los dos años de seguimiento.

craneofacial, pelvis, huesos largos, pies y manos (metacarpianos y falanges).

En carpo se han descrito varios casos de osteoblastoma en escafoides, ganchoso ${ }^{6-14}$, hueso grande $^{15}$ y piramidal ${ }^{16}$ El diagnóstico de osteoblastoma en carpo es difícil por la vaguedad de los síntomas y, sobre todo, de la radiología ${ }^{17,18}$, por lo que debe realizarse el diagnóstico diferencial con otros procesos ${ }^{19}$ : a.- según el debut de los síntomas: sinovitis extensores, síndrome túnel carpiano, fracturas, dolor regional complejo... b.- con otros tumores: quiste óseo aneurismático, tumor de células gigantes, osteosarcoma, osteoma osteoide ${ }^{20}$ y enfermedad Paget localizada.

En el caso que nos ocupa, se realizó diagnóstico diferencial entre sinovitis villonodular pigmentada, sarcoma sinovial de bajo grado y osteomielitis. Al ser insuficiente la muestra obtenida en la biopsia inicial por PAAF guiada por ecografía, se realizó artrotomía con biopsia intraoperatoria. Una vez descartada malignidad o infección se decidió realizar un raspado exhaustivo y artrodesis. Se completó el tratamiento mediante artrodesis hueso grande-ganchoso aprovechando su disposición anatómica ocupando la misma hilera carpiana para así evitar pérdidas importantes del arco de movilidad.

El tratamiento más habitualmente encontrado en la literatura consiste en la escisión en bloque o raspado y relleno con injerto óseo ${ }^{21}$ y ocasionalmente artrodesis parciales. Algunos autores han realizado cirugías de resección agresivas con reconstrucción del defecto mediante injerto óseo vascularizado ${ }^{11}$.

Cuando exista duda, crecimiento rápido y tumor de gran tamaño se recomienda realizar biopsia inicial.

La tasa de recidivas oscila entre $5-20 \%{ }^{1}$ y se relaciona con un curetaje incompleto, o con el osteoblastoma agresivo. En nuestro caso no hemos hallado recidivas en los controles clínicoradiológicos realizados.

\section{CONCLUSIONES}

El osteoblastoma es un tumor óseo benigno que raramente afecta los huesos del carpo.

La poca frecuencia de esta localización y la inespecificidad de síntomas y radiología hacen de su diagnóstico un desafío para el cirujano. El retraso diagnóstico y el tipo de tratamiento, muchas veces artrodesis parciales, provocan gran incapacidad.

\section{BIBLIOGRAFÍA}

1. Hsu CS, Hentz VR, Yao J. Tumours of the hand. Lancet Oncol, 2007; 8: 157-66.

2. Dahlin DC, Johnson EW. Giant osteoid osteoma. J Bone Joint Surg Am, 1954; 36: 559-72.

3. Lichtenstein L. Benign osteoblasma. A category of osteoid and bone forming tumors other than classical osteoid osteo- ma, which may be mistaken for giant cell tumor or osteogenic sarcoma. Cancer, 1956; 9:1044-50.

4. Jaffe HL. Benign osteoblastoma. Bull Hosp Joint Dis, 1956; 17: 141-51.

5. Muñoz-Ledesma J, De las Heras J. Osteoblastoma del hueso ganchoso. A propósito de un caso. Rev Iberoamer Cir Mano, 2009: 37: 133-7.

6. Meade RA, Allende CA, Tsai TM. Osteoblastoma of the scaphoid: a case report. J Surg Orthop Adv, 2005; 14: 125-8.

7. Xarchas KC, Leviet D. Osteoblastoma of the carpal scaphoid frequency and treatment. Acta Orthop Belg, 2002; 68: 532-6. 
8. Maréchal E. Ostéoblastome agressif du scaphoïde carpien. Rev Chir Orthop Reparatrice Appar Mot. 2000; 86: 851.

9. Ragois P, Leclerc P, Hallonet D. Ostéoblastome agressif du scaphoïde carpien. Rev Chir Orthop, 2000; 86: 94-7.

10. Maréchal E. Recurrent osteoblastoma of the hamate bone. J Hand Surg Br, 1999; 24: 757-8.

11. Van Dijk M, Winters HA, Wuisman PI. Recurrent osteoblastoma of the hamate bone. A two-stage reconstruction with a free vascularized iliac crest flap J Hand Surg Br, 1999; 24: 501-5.

12. Castelló JR, Garro L, San Miguel P, Campo M. Osteoblastoma of the scaphoid--long-term results following curettage: a case report. J Hand Surg Am, 1996; 21: 426-7.

13. Chaise F, Witvoet J. Benign osteoblastoma of the hand. A case-review of the literature. Ann Chir Main, 1983; 2: 270-2.

14. Van Giffen N, De Smet L. Osteoblastoma of the proximal ulna, an unusual cause of ulnar wrist pain. A case report. Acta Orthop Belg, 2005; 71: 736-9.

15. Irisarri C, Capomassi M. Tumores óseos benignos. En: Tumores de la Mano, Cap 7. Madrid: Ed. Méd. Panamericana, 2009: 65-83.

16. MacKenney E, Sebastián P, Ruiz J, Pérez F. Osteoblastoma benigno del piramidal. Rev Esp Cir Mano, 1991; 42: 85-8.

17. Adler CP. Multifocal osteoblastoma of the hand. Skeletal Radiol, 2000; 29: 601-4.
18. Resnick D. Huesos y articulaciones en imagen. En: Tumores y lesiones seudotumorales: imágenes y anatomía patológica de las lesiones específicas. $2^{a}$ Ed. Madrid: Elsevier. 2001: 999-1000.

19. Sforzo CR, Scarborough MT, Wright TW. Bone-forming tumors of the upper extremity and Ewing's sarcoma. Hand Clin, 2004; 20: 303-15, vi.

20. Atesok KI, Alman BA. Osteoid osteoma and osteoblastoma. J Am Acad Orthop Surg, 2011; 19: 678-89.

21. Chen YC, Wang SJ, Shen $P H$, Huang GS, Lee HS, Wu SS. Intraosseous ganglion cyst of the capitate treated by intralesional curettage, autogenous bone marrow graft and autogenous fibrin clot graft. J Chin Med Assoc, 2007; 70: 222-6. 